\title{
Multiple-choice learning of line-drawn facial features: II. Sex differences
}

\author{
MELVIN H. MARX \\ University of Missouri, Columbia, Missouri 65211
}

\begin{abstract}
This report adds data to complement those in a prior report (Marx, 1979) in which retention and transfer were found to be inhibited, for male but not for female subjects, when the subjects serving as observers were required to score their paired partners' responses in acquisition. The new data were obtained from two high school classes who first learned names for full line-drawn faces and then were given interpolated transfer tests on the individual features from those faces (reversing the procedure used in the first experiment). Few reliable main effects were found in these two sets of data, analyzed separately, but there were a large number of reliable interactions. The major result was that female subjects were clearly superior on transfer tests after they had observed in acquisition, whereas males were equally proficient after observation and performance.
\end{abstract}

In a multiple-choice learning experiment reported previously (Marx, 1979), it was found that male subjects observing paired partners' responses were inhibited both in acquisition and in transfer when required to score those responses. No such inhibition was found for female subjects (Marx, 1979, Figure 1).

The present report provides both an additional set of data to complement those previously obtained and a more complete account of the sex differences in both sets of data. The two high school classes utilized for the new data first learned names for fully drawn faces and then were tested for transfer on the individual features of which those faces were composed, thus reversing the procedure first used. Observer scoring was not manipulated in the new data, both classes scoring as observers.

\section{METHOD}

\section{Subjects}

A total of 29 females and 21 males were trained and tested in two high school psychology classes in the normal classroom setting. One pair of subjects was necessarily of mixed sex, the others were all of like sex. These subjects constituted Group F-f (trained on faces, tested on features).

Thanks are due Joan Girnis, psychology teacher at Naples Senior High School, Naples, Florida, for her cooperation in this experiment. Her two psychology classes were used, as part of the course work, to familiarize the students with psychological experimentation. In class periods subsequent to the collection of the data, the experiment was described and the nature of experimental psychology was generally discussed by the experimenter. I am also indebted to Kathleen Marx for assistance in the collection of data and to Monica Moore and George Seymour for assistance in the statistical analysis. This research was supported in part by the author's Research Career Award from the National Institute of Mental Health and by a grant from the U.S. Army Research Institute for the Behavioral and Social Sciences. The opinions expressed herein are those of the author and are in no way endorsed by the U.S. Army.

\section{Procedure}

The procedure was the same as that previously described (Marx, 1979) except that subjects were first shown the fully drawn faces (four female, four male) and then tested for transfer on the four individual features of each face (eyes, mouths, noses, and hairlines) and except that all observers scored their paired performers' responses as correct or incorrect.

\section{RESULTS}

The analysis of variance was 2 by 2 by 2 by 3 by 2 , with sex of subject, same or different sex of facial stimuli, and class as between-subjects variables and trials and performance/observation $(\mathrm{P} / \mathrm{O})$ as within-subjects variables. A slightly simpler analysis (no trials variable) was run for the retention test. Again, no main effect of primary interest was reliable, but there were a number of reliable interactions ( $p=.05$ or better). In the following presentation of results, the analyses of data from Group F-f will be compared with those from Group f-F without any further indication of the prior report of the latter data (Marx, 1979).

\section{Transfer and $\mathrm{P} / \mathrm{O}$}

For the f-F group, sex of subject interacted with $\mathrm{P} / \mathrm{O}[\mathrm{F}(1,360)=3.84, \mathrm{MSe}=1.24]$. Males transferred at approximately the same level after performance (mean of 1.83 correct face identifications) and observation (corresponding mean of 1.89), but females were markedly superior in transfer under observation (2.35 compared with 1.92 mean correct identifications).

\section{Sex of Facial Stimuli and $P / 0$}

The interaction of $\mathrm{P} / \mathrm{O}$ and same or different relationship between sex of subjects and sex of facial stimulus material is next considered. For Group F-f this interaction, in retention, was reliable $[\mathrm{F}(1,42)=7.04$, $\mathrm{MSe}=.73$ ]. There was a tendency for subjects to 


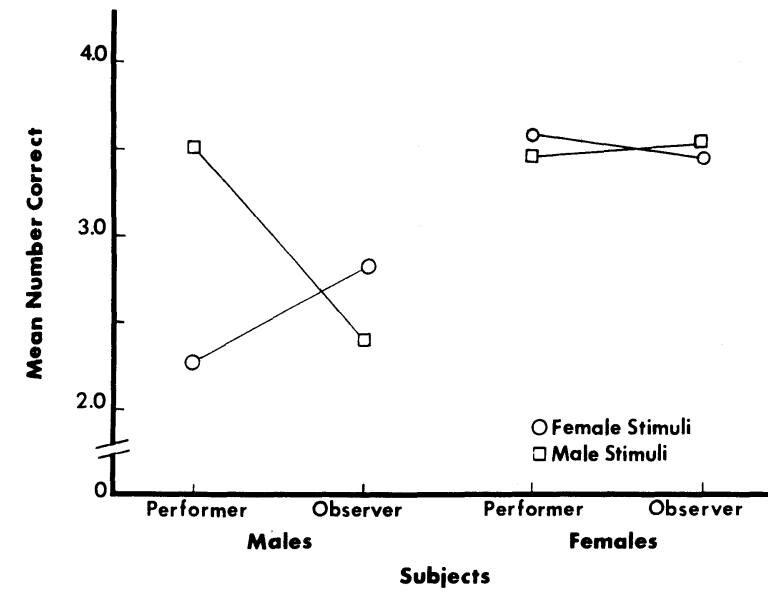

Figure 1. Mean number of features correctly identified in retention (Group f-F) as a function of sex of facial stimuli and performance or observation by male and female subjects. (Data reported in Marx, 1979.)

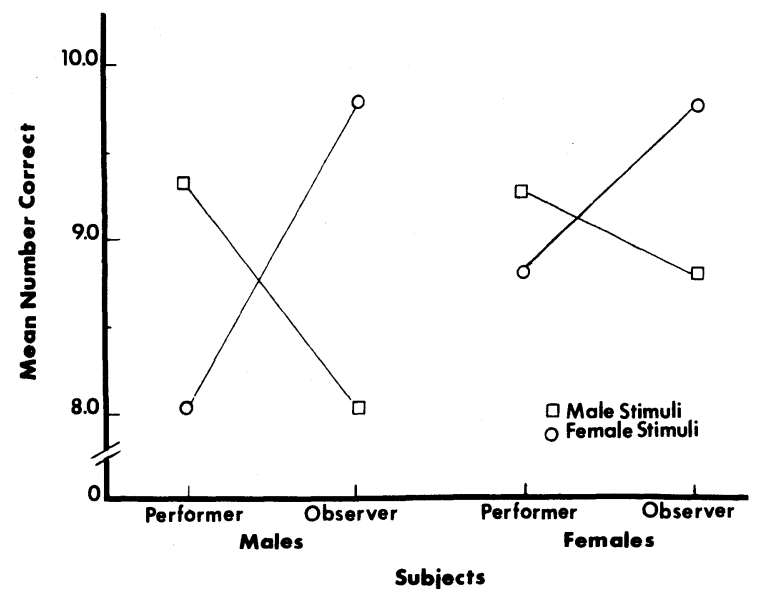

Figure 2. Mean number of faces correctly identified in retention (Group F-f) as a function of sex of facial stimuli and performance or observation by male and female subjects.

remember more same-sex faces under performance (means of 3.54 and 2.96 for same-sex and differentsex faces, respectively) and an opposite but much slighter tendency for subjects to remember more different-sex faces when observing (means of 3.23 for different-sex and 3.00 for same-sex faces). In both sets of retention data, there were also reliable interactions of $\mathrm{P} / \mathrm{O}$ and same- or different-sex facial stimuli with sex of subject $[F(1,72)=9.77, \quad \mathrm{MSe}=4.61$, for Group f-F; $F(1,42)=4.44$, MSe $=.73$, for Group F-f] . These relationships are plotted in Figures 1 and 2. In these and subsequent figures of the same type, the same-different variable has been converted to the actual sex of the stimulus materials in order to facilitate meaningful comparisons.

It is clear from these figures that male subjects were primarily responsible for the interactions. In each case males showed the same superior learning of male faces and features under performance and superior learning of

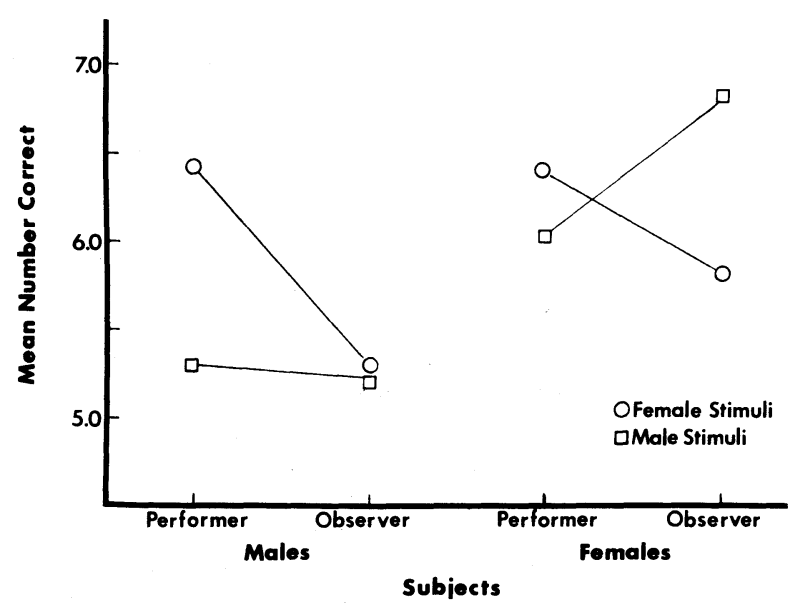

Figure 3. Mean number of faces correctly identified in transfer (Group f-F) as a function of sex of facial stimuli and performance or observation by male and female subjects. (Data reported in Marx, 1979.)

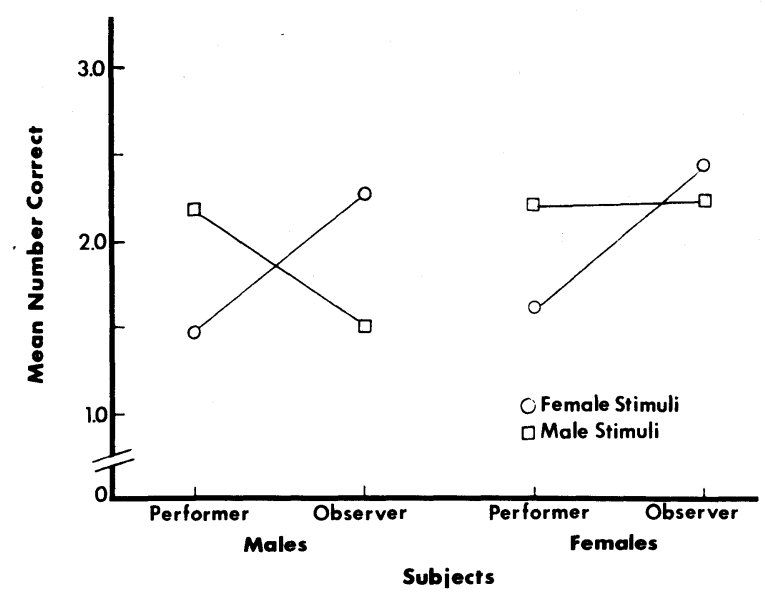

Figure 4. Mean number of features correctly identified in transfer (Group F-f) as a function of sex of facial stimuli and performance or observation by male and female subjects.

female features under observation, whereas females showed relatively slight (and opposite) tendencies of this kind.

Corresponding triple interactions $(\mathrm{P} / \mathrm{O}$ by Sex of Subject by Same-Different Stimuli) were also reliable in both f-F and F-f transfer data $[\mathrm{F}(1,72)=35.70, \mathrm{MSe}=$ 1.24 , for Group $\mathrm{f}-\mathrm{F} ; \mathrm{F}(1,42)=5.63, \mathrm{MSe}=4.99$, for Group F-f]. These interactions, plotted in Figures 3 and 4, may be compared both with each other and with the corresponding retention interactions plotted in Figures 1 and 2.

With respect to the intrinsic implications of Figures 3 and 4 , it seems that much of the effect may be attributed to the female facial stimuli. For both male and female subjects, transfer for female stimuli was clearly superior after observation when the transfer was from features to faces, and it was equally more pronounced after performance when transfer was from faces to features. No such clear pattern was shown for male stimuli, but 
there was instead a slight tendency for male subjects to transfer more after performance and for female subjects to do so after observation.

Reference to Figure 1 will indicate the much greater retention of female features, by both male and female subjects, under observation, and the comparably marked difficulty in retention under performance. Apparently, both sexes found it easier to remember and to transfer female features under observation. Male subjects, who showed a more pronounced tendency to remember male features under performance (Figure 1), likewise showed a greater tendency to transfer to male faces after performance (Figure 3).

Figures 2 and 4 indicate that this clear relationship did not hold between acquisition of faces and transfer to features. Rather, the marked superiority in retention of male faces by male subjects under performance was accompanied by only a slightly superior transfer to features, and an inconsistent relationship occurred for female stimuli. The mild interaction evident for female subjects in retention was matched by a consistent but comparably slight interaction in transfer.

\section{DISCUSSION}

Direct evidence is available in this study for the prominent role of sex differences. For example, female subjects were generally more effective as observers than as performers (see Marx, 1979, Figure 1), as has been reported previously as early as grade school (Marx \& Marx, 1976). Such differences are important in view of the recent trend to downgrade sex differences (e.g., Maccoby \& Jacklin, 1974; but see also Block, 1976).

These results, which extend the sex-difference variable to the high school population, also extend it in terms of its interaction with the score/no-score manipulation. As Figure 1 of the previous report (Marx, 1979) indicates, female students were unaffected by the scoring operation, whereas male subjects who scored as observers were generally inhibited in their transfer efforts, for $\mathbf{P}$ as well as for $\mathrm{O}$ items. The latter point is especially impressive, because it indicates considerable generalization of the inhibitory influence of the scoring operation (that is, males scored only on the items they observed, but when they did score they also had considerable difficulty transferring performance items). The data also suggest comparable difficulty in training under scoring conditions, as measured by the final retention test, which was again due primarily to male subjects.

The fact that male subjects in both groups retained markedly more correct responses for male stimuli under performance and for female stimuli under observation (Figures 1 and 2) is also interesting, albeit difficult to interpret. Whether the interaction was due at least in part to the same- or differentsex relationship or was simply due to some differential difficulty in the materials, of the sort discussed above, cannot be determined from these data alone. Also, why female subjects did not show such a clear picture is not evident. Sex differences of an apparently related sort have been found in earlier research: for example, the finding that males tend to repeat their own (performed) errors more than do females (Marx, Witter, \& Farbry, 1973). In any event it is clear that primary responsibility for the complex interactions obtained can be attributed to male subjects, as well as to female facial stimuli (as pointed out above). Replication with new materials is, of course, necessary to determine which, if any, of the relationships were functions of the particular materials and procedures used.

\section{REFERENCES}

BLOCK, J. H. Debatable conclusions about sex differences. Review of E. E. Maccoby and C. N. Jacklin, The psychology of sex differences. Contemporary Psychology, 1976, 21, 517-522.

MaccoBy, E. E., \& JaCkLIN, C. N. The psychology of sex differences. Stanford, Calif: Stanford University Press, 1974.

MarX, M. H. Multiple-choice learning of line-drawn facial features: I. Inhibitory effects of observer scoring. Bulletin of the Psychonomic Society, 1979, 14, 437-438.

MarX, M. H., \& MARX, K. Learning to spell as a function of trial-and-error performance or observation. Bulletin of the Psychonomic Society, 1976, 8, 153-155.

MarX, M. H., WitTer, D. W., \& FARBRY, J. Greater repetition of errors under performance compared to observation in multiple-choice human learning. Perceptual and Motor Skills, 1973, 37, 949-950.

(Received for publication September 22, 1979.) 